\title{
Preparing for anatomy assessment with adaptive learning resources - It is going “tibia” okay!
}

\author{
Kelly Linden, Louise Ann Pemberton, Lucy Webster
}

Faculty of Science, Charles Sturt University, Australia

\begin{abstract}
Anatomy subjects are content heavy and generally have a high stakes practical examination that is difficult to prepare for outside of the laboratory. With recent advances in technology, increased class sizes and reductions in teaching time, alternative and novel teaching technologies are emerging. An adaptive online simulated practical examination was developed. This lesson was evaluated using a survey, student interviews and learning analytics. Of the 55 students who completed the survey, 92\% indicated that the simulated practical examination enhanced their learning. Ninety two percent of students reported that the lesson met their needs for flexibility and importantly, our study also confirmed that there was a positive relationship between performance in the simulated practical exam and both the actual practical examination and overall subject score. Findings from this study revealed that adaptive learning technologies show significant promise and are an effective, flexible tool for preparing students for practical exams in a challenging subject.
\end{abstract}

Keywords: Adaptive learning; Smart Sparrow; Allied health; Anatomy; Assessment. 


\section{Introduction}

An understanding of human anatomy is essential for undergraduate allied health students. Anatomy subjects are generally taught in the first two years of undergraduate allied health courses and even for students with prior knowledge in biological science, these subjects are daunting and difficult. This is because success is dependent on spending considerable time examining cadaver specimens in the laboratory to be able to recall anatomical structures which is then assessed in an end of semester practical exam. Current limitations on how much time students can spend revising anatomy in the laboratory, increased class sizes, staffing constraints and increasing costs of cadaver-based instruction, have led to developments in online technologies. Computer based learning resources have become commonplace in the anatomy curriculum (Green et al., 2018) as evidence suggests that student success is maximised when a combination of pedagogical strategies are employed (Estai \& Bunt, 2016). Indeed, there are reports of improvements in student learning and outcomes when traditional methods are replaced with student centered learning and more interactive teaching (Biggs \& Tang, 2011; Zwick, 2018). Modern adaptive learning experiences that are designed for high engagement, yet are flexible to the needs of learners present a unique way to address challenges in teaching large, practical based anatomy subjects.

Adaptive learning personalises the student experience by adjusting the level of instruction or feedback in response to individual responses (Oxman \& Wong, 2014). It also provides a flexible learning environment where modern day students who are often time poor can control where and how they learn in an efficient manner. Adaptive learning therefore has the potential to provide genuine individualised, flexible and engaging instruction and offers a novel way to address the perceived difficulty associated with anatomy and at the same time prepare students for anatomy assessment outside of class time. One example of an adaptive learning platform is Smart Sparrow. While recent literature supports the use of Smart Sparrow across a broad range of undergraduate disciplines (Makransky et al., 2016; Polly et al., 2014; Velan, 2015; Wong, 2015), there are currently no studies specifically evaluating the use of adaptive learning technologies for teaching anatomy to allied health students and in particular how these technologies can be used to prepare students for practical assessment.

The overall aim of this study was to analyse student feedback and examination performance following completion of an online adaptive simulated practical examination. We specifically examined whether;

1. Completion of the lesson enhanced student learning in anatomy

2. Completion of the lesson provided a flexible and time efficient way of preparing for the end of session practical examination.

3. There was a relationship between performance in the simulated practical examination, the actual practical examinaiton and the overall subject score. 


\section{Methods}

The participants were 55 allied health students enrolled in first and second year anatomy subjects in 2018. The students were enrolled in the Bachelor of Podiatric Medicine, Bachelor of Occupational Therapy, Bachelor of Physiotherapy or the Bachelor of Health and Rehabilitation Science. Ethics approval for this study was received from The Charles Sturt University Human Research Ethics Committee (HREC Protocol No: 2015/265).

The Adaptive Learning platform developed by Smart Sparrow (http://smartsparrow.com) was used to create the novel adaptive simulated practical examination. A variety of high resolution photographs of cadaver specimens and anatomical models that students would be assessed on in the actual practical examination were incorporated into the lesson so that students could practice answering exam questions in an environment that was as authentic and as similar as possible to the practical exam environment (Figure 1). Adaptive feedback was built into the lesson and provided to students based on their individual responses.

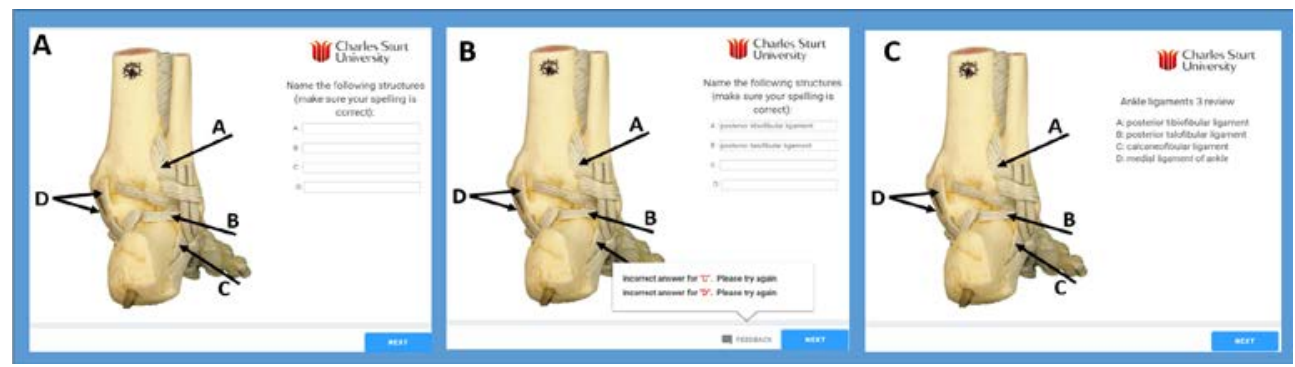

Figure 1: Screenshot of the adaptive simulated practical exam. Model with structures A-D that require labelling

(A). Feedback provided for two incorrect answers (B). Answers for all four structures displayed (C).

Students evaluated the adaptive simulated practical examination on their learning and preparation for the end of session practical examination by consenting to completing an online survey $(\mathrm{n}=55)$, making Smart Sparrow learning analytics and subject Blackboard analytics available $(n=41)$ and/or participating in an interview $(n=2)$. A structured online survey contained 6 questions measured on a 6-point Likert scale to assess the level of (i) learning from the lesson and (ii) flexibility of the lesson. In addition, students were also asked to rate their understanding of the content before and after the lesson. There were also 2 openended questions related to what the students liked most about the lesson, and what changes they would like to see to further improve the lesson. Group means were compared using a paired t-test and were analysed using the stastistical package GraphPad Prism (Version 8.0). The significance level was set at $\mathrm{p}<0.05$. For the subset of students that consented to Smart Sparrow and Blackboard data analysis, correlations between the Smart Sparrow simulated practical exam scores and overall subject score were analysed with a Pearson correlation coefficient using GraphPad Prism (Version 8.0). Significance level was set at $\mathrm{p}<0.05$. 


\section{Findings}

Overall, results from the online survey in combination with analytics revealed that the adaptive lesson facilitated learning and allowed students to prepare for the actual practical examination in a time efficient manner. In the small subset of students that agreed to the analysis of subject Blackboard data there was a positive correlation between the simulated practical test score, the actual practical test score and overall subject performance.

\subsection{Learning}

Students reported that the simulated practical examination (i) improved their learning (ii) helped to identify priorities for learning and (iii) lesson feedback enhanced their learning (Table 1). Comments from open ended questions and interviews indicated that students liked that the lesson "tested my current knowledge" and that it was in a test environment "I know you never know anything until you test yourself". Other comments relating to learning included "It did help me immensely, in my prac exam" and "My grade would have suffered I am sure, because I would have spent a lot of time trying to orientate myself with the specimen, rather than having seen it on the Smart Sparrow".

Table 1: Percentage of students that "very strongly agreed" or "strongly agreed" with statements (on a 6-point Likert Scale) relating to learning and flexibility of the simulated practical examination.

\begin{tabular}{cc}
\hline Learning & \% Agreement \\
\hline It improved my understanding of the topic & $89 \%$ \\
It helped me to identify priorities for my learning & $92 \%$ \\
It provided feedback that enhanced my learning & $83 \%$ \\
\hline Flexibility & $89 \%$ \\
\hline It made my learning more efficient (saved time) & $92 \%$ \\
It provided an individualized learning environment & $88 \%$ \\
\hline
\end{tabular}

Thirty eight students answered the question to "rate your understanding of the lesson on a scale of 1 to 10 before you used this lesson" and to "rate your understanding of the lesson on a scale of 1 to 10 after you used this lesson". Thirty two (84\%) of these students indicated that they had a better understanding of the content after completion of the lesson (5.4 vs 7.8, $\mathrm{p}<0.0001$; Figure 2). 


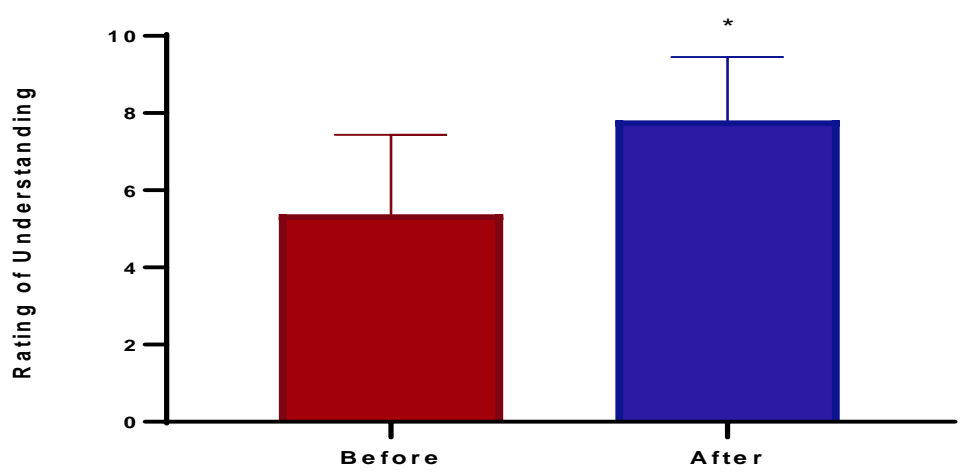

Figure 2: Improvements in students' self-reported understanding of the subject content before and after completing the simulated practical examination $* p<0.0001$.

\subsection{Flexibility and preparedness for assessed practical exam}

Students overwhelmingly reported that the simulated practical exam made learning more efficient by saving them time, met their needs for flexibility in learning and provided an individualised learning environment (Table 1).

Student feedback from the open-ended questions in the online survey and from the interviews also confirmed that the simulated practical exam provided a flexible learning environment: "It provided the framework to test yourself repeatedly" (Survey feedback) and "I wish we had these opportunities in all of our subjects" (Student interview)

A positive correlation between student performance in the simulated practice examination and performance in both the end of session practical exam $(r=0.6 ; p=<0.0001)$, and overall subject performance $(r=0.43 ; p=0.006$; Figure 3 ) also provided evidence that the adaptive lesson prepared students for assessment items. 

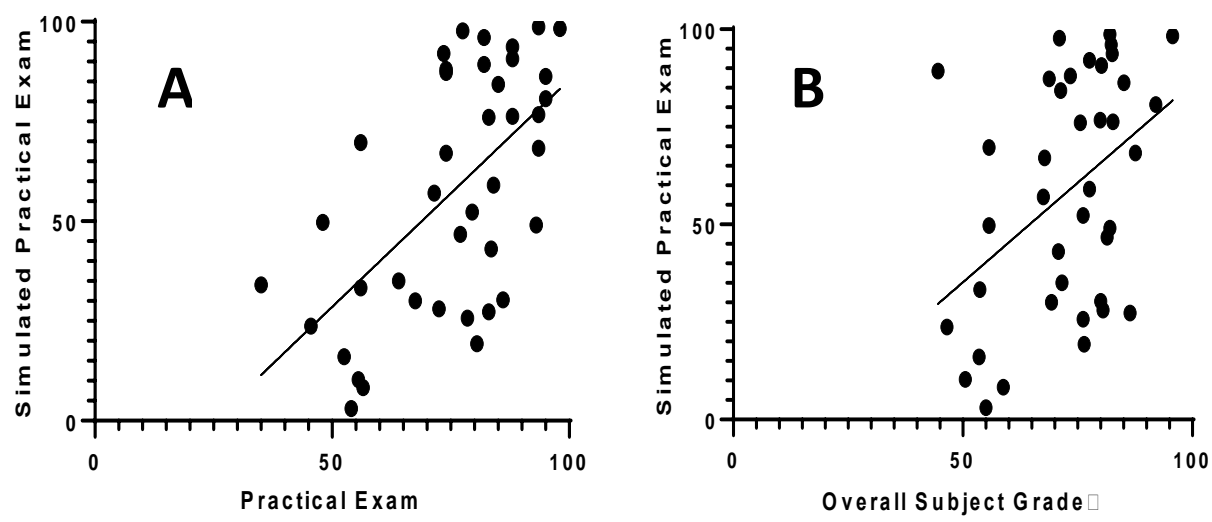

Figure 3:Correlation between performance in the simulated practical exam and the practical exam (A) and the overall subject grade.(B)

\section{Discussion and Conclusion}

Overall, this study demonstrated that completion of the adaptive lesson enhanced student learning in anatomy, provided a flexible and time efficient way of preparing for the end of session practical exam and showed that there was a relationship between performance in the simulated practical exam, the actual practical exam and the overall subject score. Our finding that the adaptive lesson enhanced student learning anatomy is consistent with other studies that have examined learning with adaptive learning resources versus conventional teaching methods (Wong, 2015; Samulski et al., 2016)

One of the most powerful findings from this study was that the simulated practical exam assisted students in preparing for the assessed practical exam by identifying gaps in their knowledge. At the same time students found the adaptive lesson to be a time efficient way of studying. So for many modern day students who often have minimal time for study, this resource is a fantastic asset - not only do students have a way of finding out what they don't know but they also have a flexible resource that they can access anytime and anywhere to consolidate that knowledge (Collis et al., 1997). These adaptive resources are not only invaluable in anatomy subjects as we have shown here but there is enormous potential for them to be applied to many different disciplines to assist students in assessment preparation particularly when students often have four end of session assessment items due at roughly the same time (Makransky et al., 2016; Green, 2018; Wong et al., 2015). Indeed, this was evident from the student interviews where there was a clear message from students wishing that the resources were available in all subjects. 
The study also demonstrated that there was a correlation between performance in the simulated practical exam and both the end of session practical exam and overall subject grade. While this was pleasing it would have been ideal to compare practical exam scores and overall subject grades between students that had completed the simulated practical examination with those that had not. This was not possible as the latter group did not give consent to access their subject analytics but future studies will aim to quantify the relationship between learning with the adaptive resource and subject success.

\section{References}

Biggs, J., \& Tang, C. (2011). Teaching for quality learning at university, $4^{\text {th }}$ Edition, Open University Press, UK.

Collis, B., Moonen, J., \& Vingerhoets, J. (1997). Flexibility as a key construct in European training: Experiences from the TeleScopia project. British Journal of Educational Technology, 28(3), 199-217. Doi:10.1111/1467-8535.00026.

Estai M, Bunt S. (2016). Best teaching practices in anatomy education: A critical review. Annals of Anatomy 208, 151-157

Green RA, Whitburn LY, Zacharias A, Byrne G, Hughes DL. (2018). The relationship between student engagement with online content and achievement in a blended learning anatomy course. Anatomical Sciences Education, 11, 471-477.

Makransky, G., Thisgaard, M,W., \& Gadegaard, H. (2016). Virtual simulations as preparation for lab exercises: Assessing learning of key laboratory skills in microbiology and improvement of essential non-cognitive skills. PLOS ONE, 11(6), 1-11. Doi:10.1371/journal.pone.0155895

Oxman, S., \& Wong, W. (2014). White paper : Adaptive learning systems. In D.X.D.E.G.I.E Solutions (Ed.): Integrated Education Solutions.

Polly, P., Marcus, N., Maguire, D., Belinson, Z., \& Velan, G. (2014). Evaluation of an adaptive virtual laboratory environment using Western Blotting for diagnosis of disease. BMC Medical Education, 14(1), 222. Doi :10.1186/1472-6920-14-222.

Samulski, T.D., La, T., Wu, R. (2016). Adaptive eLearning modules for cytopathology education: A review and approach. Diagnostic Cytopathology, 44(11). Doi: 10.1002/dc.23558.

Smart Sparrow, (2018). Adaptive learning technology. Smart Sparrow Pty Ltd., Sydney, NSW, Australia. URL:https://www.smartsparrow.com/ [accessed 20 January 2019].

Velan, G., Ben-Naim, D., Kumar, R., Bain, M., Kan, B., \& Marcus, N. (2015). Adaptive tutorials using virtual slides to enhance learning of microscopic morphology. Paper presented at the E-Learn, Kona, Hawaii, USA.

Wong, V., Smith, A., Hawking, N., Kumar, R., Young, N., Kyaw, M., \& Velan, G. (2015). Adaptive tutorials versus web-based resources in radiology: A mixed methods comparison of efficacy and student engagement. Academic Radiology, 22(10), 12991307. 
Preparing for anatomy assessment with adaptive learning resources - It is going “tibia” okay!

Zwick, M. (2018). The design, implementation and assessment of an undergraduate neurobiology course using a project-based approach. The Journal of Undergraduate Neuroscience Education (JUNE), 16(2), A131-A142. 